\title{
Effects of DLX3 on the osteogenic differentiation of induced pluripotent stem cell-derived mesenchymal stem cells
}

\author{
JUNYUAN LI $^{1 *}$, QIANG LIN ${ }^{1 *}$, YIXIN LIN $^{1}$, RENFA LAI $^{1}$ and WEN ZHANG ${ }^{2,3}$ \\ ${ }^{1}$ The Medical Center of Stomatology, The First Affiliated Hospital of Jinan University, Guangzhou, Guangdong 510630; \\ ${ }^{2}$ Department of Endodontics, Guanghua School of Stomatology, Hospital of Stomatology, Sun Yat-sen University, Guangzhou, \\ Guangdong 510055; ${ }^{3}$ Department of Oral Digital Center, Stomatology Hospital of Guangzhou Medical University, \\ Guangzhou, Guandong 510140, P.R. China
}

Received May 3, 2020; Accepted December 2, 2020

DOI: $10.3892 / \mathrm{mmr} .2021 .11871$

\begin{abstract}
Osteoporosis is a disease characterized by the degeneration of bone structure and decreased bone mass. Induced pluripotent stem cell-derived mesenchymal stem cells (iPSC-MSCs) have multiple advantages that make them ideal seed cells for bone regeneration, including high-level proliferation, multi-differentiation potential and favorable immune compatibility. Distal-less homeobox (DLX)3, an important member of the DLX family, serves a crucial role in osteogenic differentiation and bone formation. The present study aimed to evaluate the effects of DLX3 on the proliferation and osteogenic differentiation of human iPSC-MSCs. iPSC-MSCs were induced from iPSCs, and identified via flow cytometry. Alkaline phosphatase (ALP), Von Kossa, Oil Red $\mathrm{O}$ and Alcian blue staining methods were used to evaluate the osteogenic, adipogenic and chondrogenic differentiation of iPSC-MSCs. DLX3 overexpression plasmids were constructed and transfected into iPSC-MSCs to generate iPSC-MSC-DLX3. iPSC-MSC-GFP was used as the control. Reverse transcription-quantitative PCR (RT-qPCR) and western blotting were performed to measure the expression of DLX3 2 days after transfection. Subsequently, cell proliferation was assessed using a Cell Counting Kit-8 assay on days 1, 3, 5 and 7. RT-qPCR and western blotting were
\end{abstract}

Correspondence to: Professor Renfa Lai, The Medical Center of Stomatology, The First Affiliated Hospital of Jinan University, 613W Huangpu Avenue, Guangzhou, Guangdong 510630, P.R. China E-mail: 13318818388@163.com

Dr Wen Zhang, Department of Endodontics, Guanghua School of Stomatology, Hospital of Stomatology, Sun Yat-sen University, 56 Lingyuan West Road, Guangzhou, Guangdong 510055, P.R. China E-mail: northmoss@126.com

*Contributed equally

Key words: distal-less homeobox 3, proliferation, osteogenic differentiation, induced pluripotent stem cell-derived mesenchymal stem cells used to analyze osteogenic-related gene and protein expression levels on day 7. ALP activity and mineralized nodules were assessed via ALP staining on day 14. Statistical analysis was performed using an unpaired Student's t-test. Flow cytometry results demonstrated that iPSC-MSCs were positive for CD73, CD90 and CD105, but negative for CD34 and CD45. iPSC-MSC-DLX3 had significantly lower proliferation compared with iPSC-MSC-GFP on days 5 and $7(\mathrm{P}<0.05)$. mRNA expression levels of osteogenic markers, such as ALP, osteopenia (OPN), osteocalcin (OCN) and Collagen Type I (COL-1), were significantly increased in iPSC-MSC-DLX3 compared with iPSC-MSC-GFP on day $7(\mathrm{P}<0.05)$. Similarly, the protein expression levels of ALP, OCN, OPN and COL-1 were significantly increased in iPSC-MSC-DLX3 compared with iPSC-MSC-GFP on day $7(\mathrm{P}<0.05)$. The number of mineralized nodules in iPSC-MSC-DLX3 was increased compared with that in iPSC-MSC-GFP on day $14(\mathrm{P}<0.05)$. Thus, the present study demonstrated that DLX3 serves a negative role in proliferation, but a positive role in the osteogenic differentiation of iPSC-MSCs. This may provide novel insight for treating osteoporosis.

\section{Introduction}

Bone is in a dynamic balance between bone formation and bone resorption (1). With aging and after the menopause, an imbalance in bone resorption relative to formation results in osteoporosis (2). Osteoporosis is a common skeletal disease characterized by decreased bone mass, deterioration of bone microarchitecture and increased susceptibility to fractures. With the gradual aging of the population in many countries, osteoporosis has become a major global health concern (3). In the United States, osteoporosis is projected to affect nearly 14 million adults over the age of 50 by the year 2020 (4). Worldwide, 200 million women have osteoporosis (5).

Skeletal development is regulated by numerous homeodomain proteins, including the distal-less (DLX) family, which play an important role in the development of bone tissue (6). DLX family has six transcription factor members, known as DLX1-6 (7). DLX3 is mapped to $17 \mathrm{q} 21.33$ and has a notable impact on the development of organs, including glands, teeth and hair follicles $(8,9)$. In humans, mutation of DLX3 can 
cause tricho-dento-osseous syndrome, which is characterized by hypoplasia of the hair, enamel and dentin, as well as a high bone density (8).

Bone marrow mesenchymal stem cells (BMSCs) are a type of post-natal stem cell with the potential to differentiate into different cells, such as osteoblasts, chondrocytes and adipocytes (10). Bone formation depends on the osteogenic potential and proliferative capability of BMSCs, but the proliferative ability of BMSCs is limited, which has restricted their application in clinical settings $(10,11)$. Therefore, there is a need for an improved cell source to overcome the limitations of BMSCs (11). Induced pluripotent stem cell-derived mesenchymal stem cells (iPSC-MSCs), which are an unlimited source of MSCs, exhibit favorable proliferation, cell viability, and osteo- and chondrogenic differentiation potential, and thus could be used to meet the requirement of bone regeneration (11-15). Previously, several cell culture supplements have been utilized to derive MSCs from iPSC (11). The most commonly used supplements are synthetic coatings, such as PMEDSAH (15), fibrillar collagen (16), extracellular matrix Geltrex (17) and fibronectin (17). Moreover, a few small molecules, such as SB431542 (TGF- $\beta$ pathway inhibitor) (18) and CHIR99021 (GSK inhibitor) (19) have been applied to obtain iPSC-MSCs. In the current study, iPSC-MSCs were derived using Geltrex, which retains the complete osteogenesis function of the cells $(12-14,17)$.

A previous study revealed that overexpression of DLX3 enhances the osteogenic differentiation of human BMSCs (20). Similar results have also been reported in human dental pulp cells (DPCs), in which overexpressed DLX3 decreases cell proliferation and increases mRNA expression levels of osteo- and chondrogenic markers, including alkaline phosphatase (ALP), dentin sialophosphoprotein (DSPP) and dentin matrix acidic phosphoprotein 1 (DMP1) (21). Thus, the DLX3 gene is crucial for osteogenic differentiation. However, the role of DLX3 gene in regulating iPSC-MSCs is yet to be fully elucidated.

In the present study, DLX3-overexpression iPSC-MSCs (iPSC-MSC-DLX3) were constructed, the expression of DLX3 in iPSC-MSCs and iPSC-MSC-DLX3 was examined by reverse transcription-quantitative PCR (RT-qPCR) and western blotting, and then the effects of the DLX3 gene on osteogenic differentiation of these cells was evaluated.

\section{Materials and methods}

Derivation of iPSC-MSCs. The experiment protocol of the present study was approved by the Ethical Review Committee of Jinan University (approval no. 2015-045). Human iPSC line was obtained from the South China Institute for Stem Cell Biology and Regenerative Medicine Group of the Chinese Academy of Sciences. iPSCs were cultured in mTeSR1 medium (Stemcell Technologies, Inc.) on Geltrex ${ }^{\mathrm{TM}}$ LDEV-Free Reduced Growth Factor Basement Membrane Matrix (Gibco; Thermo Fisher Scientific, Inc.) coated dishes for 5 days. Then, the medium was replaced with DMEM (Gibco; Thermo Fisher Scientific, Inc.) containing 10\% FBS (Gibco; Thermo Fisher Scientific, Inc.), 2 mM L-glutamine, $1 \%$ penicillin/streptomycin and $0.1 \mathrm{mM}$ non-essential amino acids (Gibco; Thermo Fisher Scientific, Inc.). When these cells reached $80 \%$ confluence, they were passaged. When most of the cells presented spindle-like morphology, they were collected and applied for subsequent experiments.

Flow cytometry analysis. Flow cytometry was used to evaluate surface markers of iPSC-MSCs. Briefly, the cells were harvested, and then 3\% BSA (Gibco; Thermo Fisher Scientific, Inc.) was used to block non-specific antigens on the cell surface at $37^{\circ} \mathrm{C}$ for $30 \mathrm{~min}$. Subsequently, the cells were incubated with monoclonal antibodies (all purchased from BioLegend, Inc.) against CD73 (cat. no. 344005), CD90 (cat. no. 328113), CD105 (cat. no. 323207), CD34 (cat. no. 343607) and CD45 (cat. no. 368511). Then, the cells were washed three times with BSA to remove non-specific antibodies. A Guava ${ }^{\circledR}$ easyCyte ${ }^{\mathrm{TM}}$ flow cytometer and Guava ${ }^{\circledR}$ Suite Software 3.4 (both EMD Millipore) were used to analyze surface antigens.

Differentiation capability analysis. The osteogenic, adipogenic and chondrogenic differentiation capabilities of iPSC-MSCs were analyzed. To study osteogenic differentiation, iPSC-MSCs at $1 \times 10^{4} /$ well density were seeded on 6-well plates (Corning, Inc.) and incubated with $2 \mathrm{ml} \mathrm{StemPro}{ }^{\mathrm{TM}}$ Osteogenesis Differentiation medium (Gibco; Thermo Fisher Scientific, Inc.) at $37^{\circ} \mathrm{C}$ for 3 weeks. The Osteogenesis Differentiation medium was replaced every 3 days. The cells were fixed with $4 \%$ paraformaldehyde for $30 \mathrm{~min}$ at room temperature. The ALP activity of iPSC-MSCs was assayed using an ALP staining kit (Sigma-Aldrich; Merck KGaA) according to the manufacturer's instructions, and the calcified matrix deposition was detected using a Von Kossa staining kit (Nanjing Jiancheng Bioengineering Institute) according to the manufacturer's instructions and observed under a light microscope (Zeiss Axio Observer.Z1; Carl Zeiss AG).

In order to study adipogenic differentiation, iPSC-MSCs at $1 \times 10^{4} /$ well density were seeded on 6-well plates and incubated with $2 \mathrm{ml} \mathrm{StemPro}{ }^{\mathrm{TM}}$ Adipogenesis Differentiation medium (Gibco; Thermo Fisher Scientific, Inc.) at $37^{\circ} \mathrm{C}$ for 3 weeks. Adipogenesis Differentiation medium was replaced every 3 days, and subsequently the cells were assayed with an Oil Red O kit (Nanjing Jiancheng Bioengineering Institute) for $15 \mathrm{~min}$ at room temperature.

To study chondrogenic differentiation, iPSC-MSCs at $1 \times 10^{4} /$ well density were seeded on 6-well plates and incubated with $2 \mathrm{ml} \mathrm{StemPro}{ }^{\mathrm{TM}}$ Chondrogenesis Differentiation medium (Gibco; Thermo Fisher Scientific, Inc.) at $37^{\circ} \mathrm{C}$ for 3 weeks. The Chondrogenesis Differentiation medium was replaced every 3 days, and subsequently the cells were assayed with an Alcian blue kit (Nanjing Jiancheng Bioengineering Institute) for $30 \mathrm{~min}$ at room temperature.

Lentiviral plasmid transfection. Human DLX3 gene primers (PrimerBank ID:38327640c1, Table I) were designed according to PrimerBank online (The Massachusetts General Hospital) (22). Subsequently, PCR amplification was performed according to our previous study (23). The amplified DLX3 primers were treated with EcoRI and BamHI, and then combined into the lentivirus vector pCDH-CMV-MCS-EF1-copGFP (pCDH; System Biosciences, LLC) to construct the recombinant plasmid pCDH-DLX3. The target gene was transduced into $4 \times 10^{5}$ 293FT cells per 
well in 6 well plate using a combination of enveloping plasmid, packaging plasmid and recombinant lentiviral plasmid (3rd generation system; Cyagen Biosciences, Inc.) at $37^{\circ} \mathrm{C}$. Then, $48 \mathrm{~h}$ later, 293FT cells were centrifuged at $10,000 \mathrm{x} \mathrm{g}$ at $4^{\circ} \mathrm{C}$ for $4 \mathrm{~h}$ and the supernatant was collected to infect the 3rd passage of iPSC-MSCs (iPSC-MSC-DLX3) at $37^{\circ} \mathrm{C}$. The MOI was 50 , and concentration of Polybrene was $5 \mu \mathrm{g} / \mathrm{ml}$. Cells were cultured at $37^{\circ} \mathrm{C}$ in DMEM containing $10 \%$ FBS with $1 \mu \mathrm{g} / \mathrm{ml}$ puromycin for 3 days in order to select puromycin-resistant cells and $0.25 \mu \mathrm{g} / \mathrm{ml}$ puromycin was used for maintenance. Similarly, iPSC-MSCs transfected with blank lentivirus vector (iPSC-MSC-GFP) were generated and used as the control. After 2 days, reverse transcription-quantitative PCR (RT-qPCR) and western blot analysis were performed to evaluate the expression of DLX3 in the two groups.

Cell proliferation assay. Proliferation of iPSC-MSC-GFP and iPSC-MSC-DLX3 was assessed using Cell Counting Kit-8 (CCK-8; Dojindo Molecular Technologies, Inc.). On the first day, cells were seeded on 96 -well plates at $2 \times 10^{3}$ cells/well, and then cultured with DMEM plus 10\% FBS. Cell viability was evaluated on day 1, 3, 5 and 7 post-transfection. Optical density (OD) at $450 \mathrm{~nm}$ was recorded using an enzyme immunoassay reader (Bio-Rad Laboratories, Inc.).

$R T-q P C R$ analysis. Total RNA was isolated from iPSC-MSC-GFP and iPSC-MSC-DLX3 using TRIzol ${ }^{\circledR}$ reagent (Invitrogen; Thermo Fisher Scientific, Inc.) on day 7. NanoDrop $^{\mathrm{TM}} 2000$ system (Thermo Fisher Scientific, Inc.) was used to test RNA concentrations. The extracted RNA was reverse-transcribed into cDNA using iScript ${ }^{\mathrm{TM}}$ gDNA Clear cDNA Synthesis kit (Invitrogen; Thermo Fisher Scientific, Inc.) following the manufacturer's instructions. qPCR analysis was operated using PowerUp SYBR-Green Master Mix (Invitrogen; Thermo Fisher Scientific, Inc.) and measured by spectrofluorimetric iQ5 Thermal iCycler (Bio-Rad Laboratories, Inc.) according to the manufacturer's instructions. The primer sequences of DLX3, ALP, osteopontin (OPN), osteocalcin (OCN), Collagen Type I (COL-1) and GAPDH are presented in Table I. The relative expression of target genes was determined using the $2^{-\Delta \Delta \mathrm{Cq}}$ method (24) and normalized to GAPDH. Each sample was tested three times.

Western blot analysis. A total of 7 days after transfection, iPSC-MSC-GFP and iPSC-MSC-DLX3 were rinsed with PBS and lysed in $0.1 \mathrm{ml}$ RIPA buffer containing $10 \mathrm{mg} / \mathrm{ml}$ proteinase inhibitor PMSF (Invitrogen; Thermo Fisher Scientific, Inc.) on ice for $30 \mathrm{~min}$. The lysed cells were centrifuged at $10,000 \mathrm{x} \mathrm{g}$ for $10 \mathrm{~min}$ at $4^{\circ} \mathrm{C}$, and the supernatant was collected. During electrophoresis, $20 \mu \mathrm{g}$ target total protein/lane was separated via SDS-PAGE on a $10 \%$ gel (Beyotime Institute of Biotechnology), which were subsequently transferred to PVDF membranes (Thermo Fisher Scientific, Inc.) at $200 \mathrm{~mA}$ for $2 \mathrm{~h}$. PVDF membranes were blocked using 5\% non-fat milk with TBS with $0.1 \%$ Tween-20 at room temperature for $2 \mathrm{~h}$, and then incubated with the primary antibodies at $4^{\circ} \mathrm{C}$ overnight. The primary antibodies used were as follows: DLX3 (1:500; cat. no. ab64953; Abcam), ALP (1:500; cat. no. ab83259; Abcam), OPN (1:500; cat. no. ab8448; Abcam), OCN (1:500; cat. no. ab93876; Abcam), COL-1 (1:500; cat.
Table I. Primers used for reverse transcription-quantitative PCR.

\begin{tabular}{llc}
\hline Gene & \multicolumn{1}{c}{ Primer sequences $\left(5^{\prime} \rightarrow 3^{\prime}\right)$} & Size $(\mathrm{bp})$ \\
\hline DLX3 & $\begin{array}{l}\text { F: TACCCTGCCCGAGTCTTCTG } \\
\text { R: TGGTGGTAGGTGTAGGGGTTC }\end{array}$ & 111 \\
ALP & $\begin{array}{l}\text { F: ACCACCACGAGAGTGAACCA } \\
\text { R: CGTTGTCTGAGTACCAGTCCC }\end{array}$ & 79 \\
OPN & $\begin{array}{l}\text { F: CTCCATTGACTCGAACGACTC } \\
\text { R: CAGGTCTGCGAAACTTCTTAGAT }\end{array}$ & 230 \\
& $\begin{array}{l}\text { F: CACTCCTCGCCCTATTGGC } \\
\text { OCN }\end{array}$ & 112 \\
COL-1 & $\begin{array}{l}\text { F: CCCTCCTGCTTGGACACAAAG } \\
\text { GAPDH }\end{array}$ & $\begin{array}{l}\text { F: CAGATCACGTCATCGCACAAC } \\
\text { F: GGCGAGATCCCTCCAAAAT }\end{array}$ \\
& R: GGCTGTGTCATACTTCTCATGG & 197 \\
\end{tabular}

DLX3, distal-less homeobox 3; ALP, alkaline phosphatase; OPN, osteopontin; OCN, osteocalcin; COL-1, Collagen Type I; F, forward; R, reverse.

no. ab34710; Abcam) and GAPDH (1:2,500; cat. no. ab9485; Abcam). Then, PVDF membranes were incubated with a secondary antibody (1:2,500; cat. no. ab97051; Abcam) at $37^{\circ} \mathrm{C}$ for $2 \mathrm{~h}$. GAPDH was used as the control. The blotting results were visualized with chemiluminescent western blotting detection reagents (Pierce; Thermo Fisher Scientific, Inc.) and measured by Image-Pro Plus version 6.0 (Media Cybernetics, Inc.).

ALP staining and mineralized nodule counting. The transfected cells were seeded on 6-well plates at an initial density of $3 \times 10^{4}$ cells/well and cultured for 14 days to $80 \%$ confluence in DMEM containing 10\% FBS. The cells were fixed with $4 \%$ paraformaldehyde for $30 \mathrm{~min}$ at room temperature. The ALP Staining kit (Nanjing Jiancheng Bioengineering Institute) was used to stain cells for $30 \mathrm{~min}$ at room temperature on day 14 after transfection. In total, three randomized observation views were selected, and the number of mineralized nodules was counted under a microscope (Zeiss Axio Observer.Z1; Carl Zeiss AG).

Statistical analysis. SPSS 20.0 software (IBM Corp.) was used to analyze the data, which are presented as the mean \pm SD. Statistical significance was calculated using an unpaired Student's t-test. $\mathrm{P}<0.05$ was considered to indicate a statistically significant difference.

\section{Results}

Characterization of iPSC-MSCs. As illustrated in Fig. 1A, human iPSCs presented as packed clones with clear margins. After culturing in MSC medium for 14 days, iPSCs were gradually induced into MSCs. Under the microscope, borders of the colonies were eliminated (Fig. 1B), and cells exhibited homogeneous spindle-like morphology (Fig. 1C). The iPSC-derived cells were positive for CD73 (99\%), CD90 (71\%) and $\mathrm{CD} 105$ (95\%), and negative for CD34 and CD45, as determined by flow cytometric analysis (Fig. 1D). 

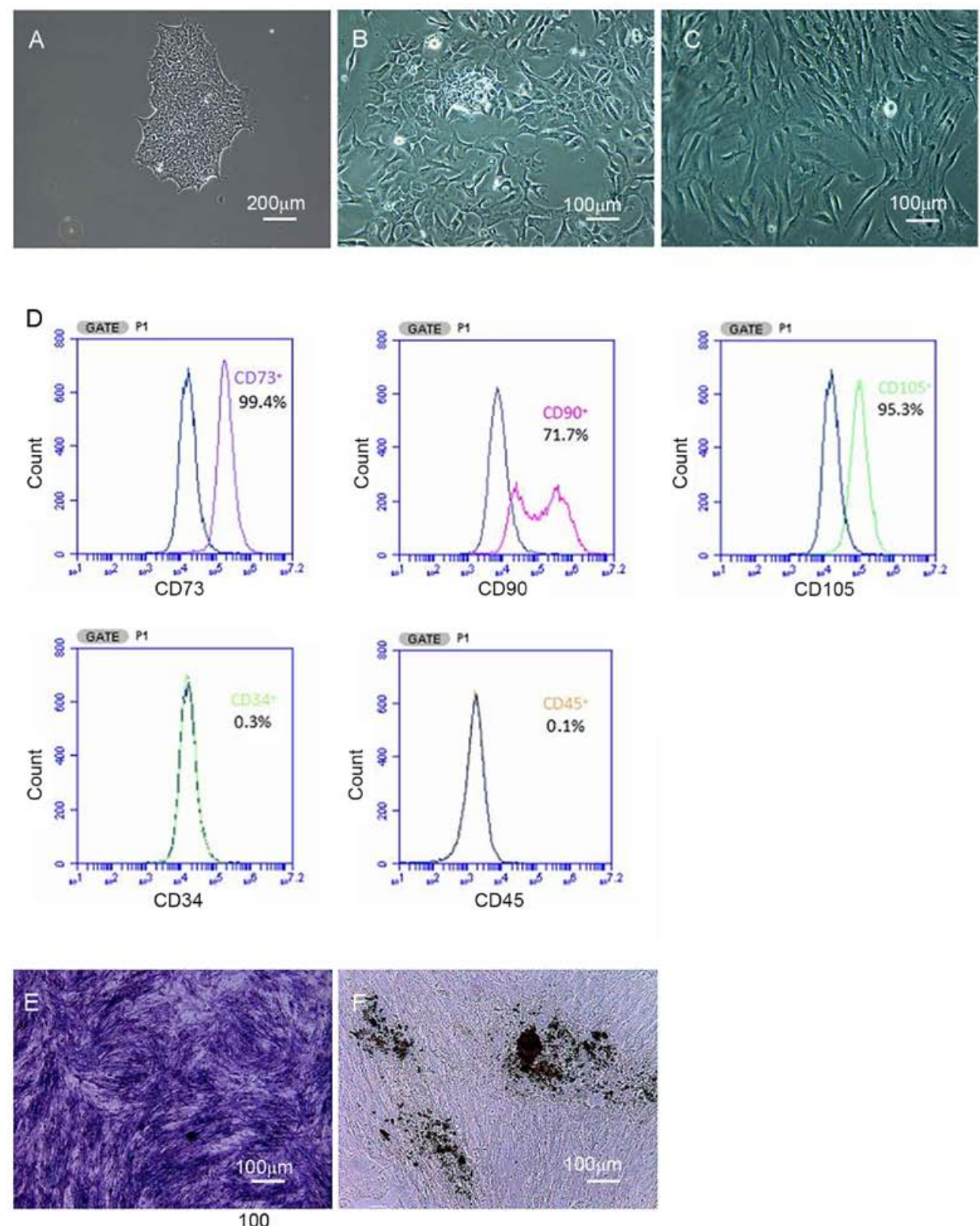

100
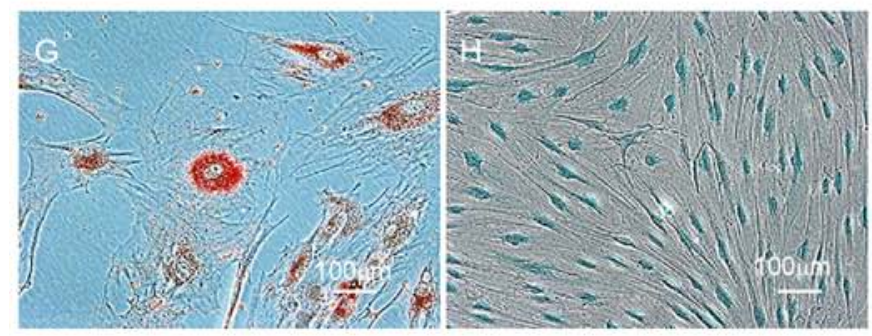

Figure 1. Generation and identification of iPSC-MSCs. (A-C) Generation of iPSC-MSCs. (A) Scale bar, $200 \mu \mathrm{m}$; (B and C) scale bar, $100 \mu \mathrm{m}$. (D) Flow cytometric analysis of iPSC-MSCs. (E) Alkaline phosphatase staining. (F) Osteogenesis was detected using Von Kossa staining. (G) Adipogenesis was detected using Oil Red O staining. (H) Chondrogenesis was detected using Alcian blue staining (scale bar, $100 \mu \mathrm{m}$ ). iPSC-MSC, induced pluripotent stem cell-derived mesenchymal stem cell.

After osteogenic induction, iPSC-derived cells displayed positive ALP staining (Fig. 1E) and Von Kossa staining (Fig. 1F). Additionally, iPSC-derived cells had positive Oil Red $\mathrm{O}$ and Alcian blue staining after adipogenic (Fig. 1G) and chondrogenic induction (Fig. 1H). Thus, iPSCs were successfully induced into iPSC-MSCs.
Expression of DLX3 in iPSC-MSCs. Both iPSC-MSC-GFP and iPSC-MSC-DLX3 groups had a GFP expression percentage of $\sim 100 \%$ (Fig. 2A). RT-qPCR results indicated that the relative mRNA expression of DLX3 in iPSC-MSC-GFP and iPSC-MSC-DLX3 was $1.00 \pm 0.05$ and $5.84 \pm 0.89$, respectively (Fig. 2B). Moreover, the western 
A

Phase contrast

GFP

Merge

iPSC-MSC-GFP
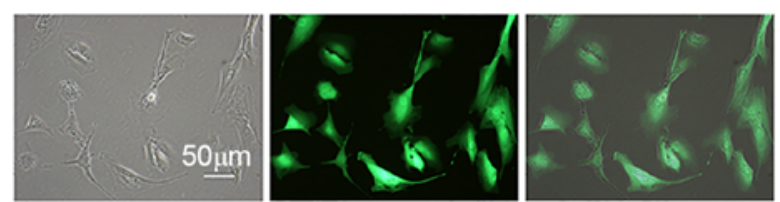

iPSC-MSC-DLX3
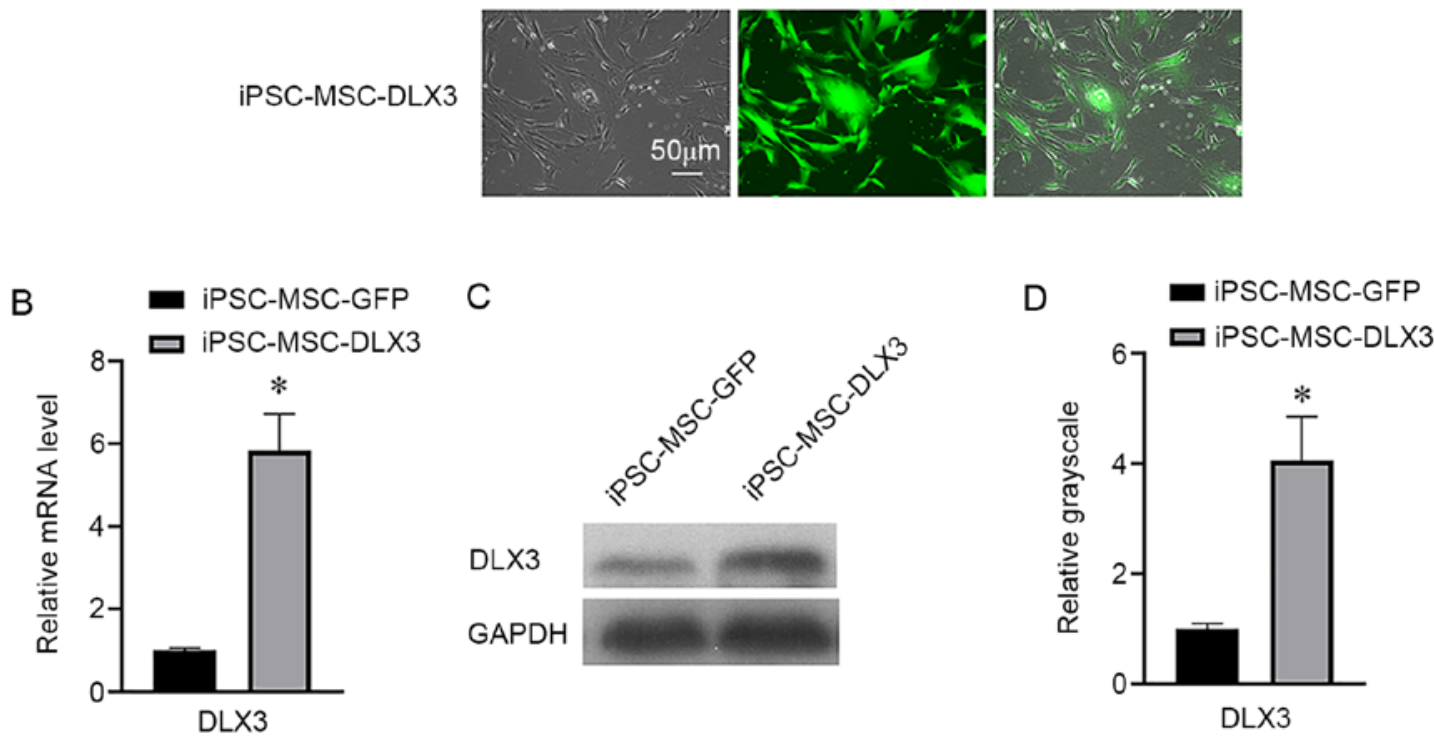

Figure 2.Lentiviral transfection efficiency. (A) GFP expression percentage in the iPSC-MSC-GFP and iPSC-MSC-DLX3 groups (scale bar, $50 \mu \mathrm{m}$ ). (B) Reverse transcription-quantitative PCR analysis of DLX3 gene expression. (C and D) Western blot analysis of DLX3 protein expression. "P<0.05 vs. iPSC-MSC-GFP. iPSC-MSC, induced pluripotent stem cell-derived mesenchymal stem cell; DLX3, distal-less homeobox 3.

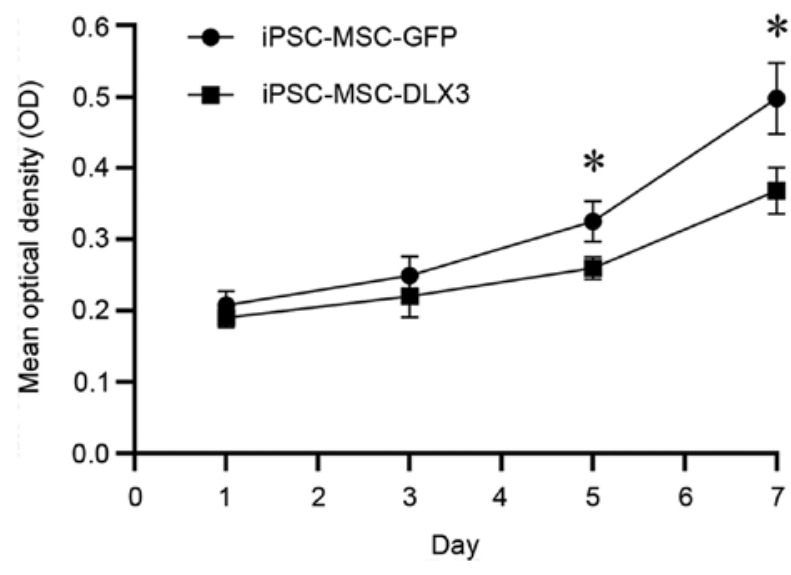

Figure 3. Cell proliferation. Cell proliferation rate was significantly reduced in the iPSC-MSC-DLX3 group compared with the rate observed intheiPSC-MSC-GFP group on days 5 and 7 . ${ }^{*} \mathrm{P}<0.05$ vs. iPSC-MSC-DLX3 iPSC-MSC, induced pluripotent stem cell-derived mesenchymal stem cell; DLX3, distal-less homeobox 3.

blotting results demonstrated that the relative DLX3 expression in iPSC-MSC-GFP and iPSC-MSC-DLX3 was $1.00 \pm 0.10$ and $4.05 \pm 0.81$, respectively (Fig. $2 \mathrm{C}$ and $\mathrm{D}$ ). Based on these results, it was suggested that the DLX3 gene was successfully transfected into iPSC-MSCs.

DLX3 regulates proliferation of iPSC-MSCs. The OD values of iPSC-MSC-GFP on day $1,3,5$ and 7 were $0.21 \pm 0.02$, $0.25 \pm 0.03,0.33 \pm 0.03$ and $0.50 \pm 0.05$, while the $\mathrm{OD}$ values of iPSC-MSC-DLX3 on day $1,3,5$ and 7 were $0.19 \pm 0.01$,
$0.22 \pm 0.03,0.26 \pm 0.02$ and $0.37 \pm 0.03$, respectively (Fig. 3). There was no significant difference in cell numbers between the two groups on days 1 and 3 ( $\mathrm{P}>0.05$ ). However, iPSC-MSC-DLX3 demonstrated significantly lower proliferative activity compared with the iPSC-MSC-GFP group on days 5 and $7(\mathrm{P}<0.05)$.

Expression of osteogenesis related genes and proteins. After 7 days of transfection of iPSC-MSC-GFP and iPSC-MSC-DLX3, the relative expression levels of ALP were $1.00 \pm 0.33$ and $11.99 \pm 0.24$, those of OPN were $1.00 \pm 0.49$ and $5.80 \pm 0.07$, those of OCN were $1.00 \pm 0.06$ and $8.64 \pm 0.11$, those of COL- 1 were $1.00 \pm 0.53$ and $5.98 \pm 0.02$. The results indicated that the mRNA expression levels of osteogenic markers in iPSC-MSC-DLX3 were significantly higher compared with those in iPSC-MSC-GFP ( $\mathrm{P}<0.05$; Fig. 4A).

Similarly, the relative expression levels of ALP were $1.00 \pm 0.13$ and $7.77 \pm 0.05$, those of OPN were $1.00 \pm 0.04$ and $2.05 \pm 0.04$, those of OCN were $1.00 \pm 0.05$ and $3.79 \pm 0.17$, and those of COL-1 were $1.00 \pm 0.11$ and $3.38 \pm 0.22$. The expression levels of the osteogenic proteins in iPSC-MSC-DLX3 were significantly increased compared with those in iPSC-MSC-GFP ( $\mathrm{P}<0.05$; Fig. 4B and $\mathrm{C})$.

ALP activity and mineralization. As presented in Fig. 5A, ALP was stained as a golden color. ALP staining in the iPSC-MSC-DLX3 group was increased and brighter compared with that in the iPSC-MSC-GFP group. Mineralized nodules were stained as black/brown in color. The number of mineralized nodules in iPSC-MSC-DLX3 was significantly higher compared with that in iPSC-MSC-GFP $(\mathrm{P}<0.05$; Fig. 5B). 
A

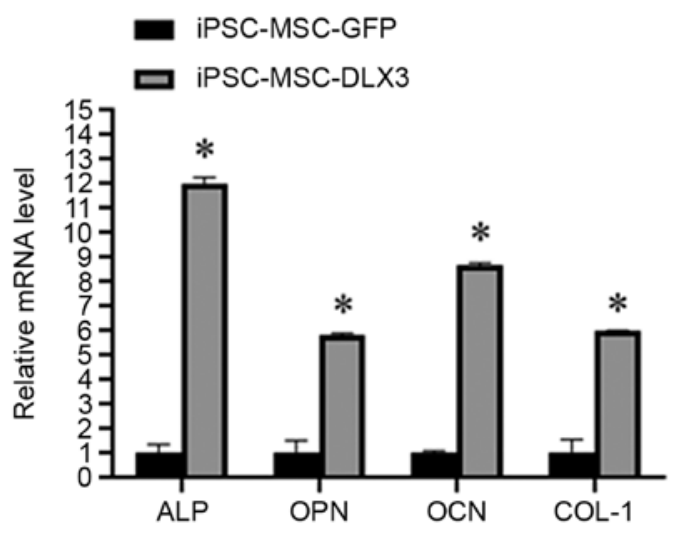

B

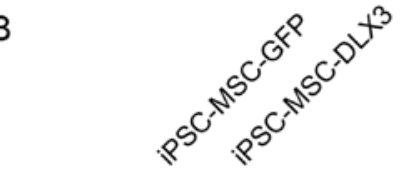

C

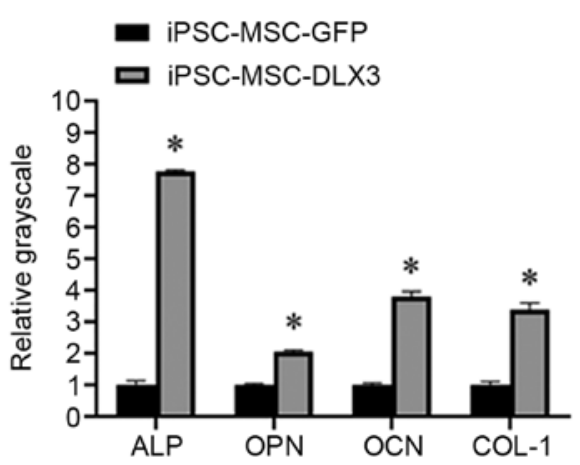

Figure 4. mRNA and protein expression of osteogenic differentiation-related genes on day 7. (A) Reverse transcription-quantitative PCR analysis of osteogenic-related gene expression. (B and C) Western blot analysis of osteogenic-related protein expression. ${ }^{*} \mathrm{P}<0.05$ vs. iPSC-MSC-GFP. iPSC-MSC, induced pluripotent stem cell-derived mesenchymal stem cell; DLX3, distal-less homeobox 3; ALP, alkaline phosphatase; OPN, osteopenia; OCN, osteocalcin; COL-1, Collagen Type I.

A

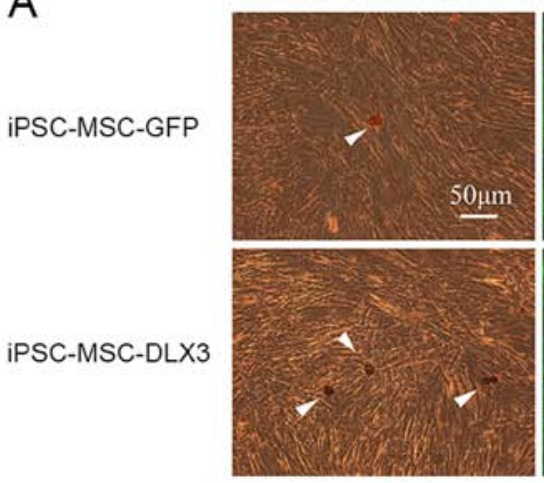

GFP

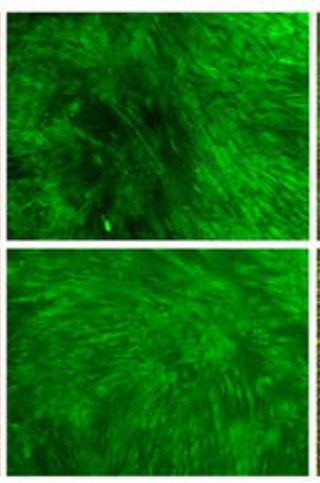

Merge

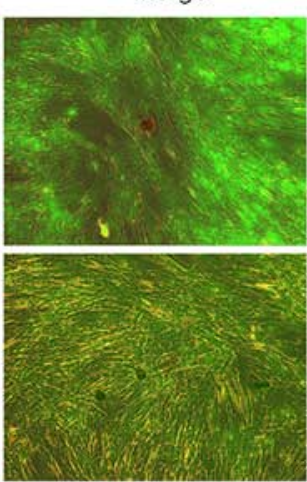

B

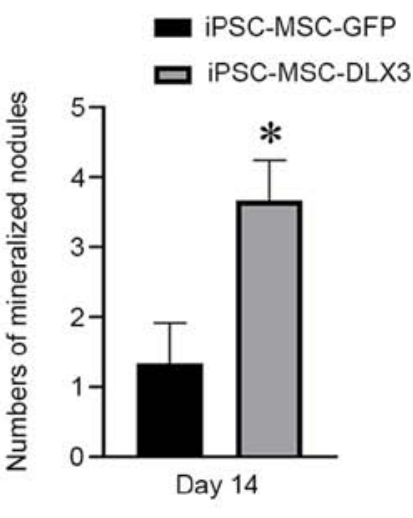

Figure 5. ALP activity and mineralized nodules. (A) ALP was stained as golden, and mineralized nodules were stained as black/brown (marked with arrow). Scale bar, $50 \mu \mathrm{m}$. (B) The number of mineralized nodules in the iPSC-MSC-DLX3 group was higher than that in the iPSC-MSC-GFP group. . P $<0.05$ vs. iPSC-MSC-GFP. iPSC-MSC, induced pluripotent stem cell-derived mesenchymal stem cell; DLX3, distal-less homeobox 3; ALP, alkaline phosphatase.

\section{Discussion}

iPSC-MSCs have been considered as a novel cell resource for bone tissue engineering (11-14,17). The present study used Geltrex as a cell culture coating to generate MSCs from iPSCs, as Geltrex can enhance cell attachment and improve the efficacy to generate iPSC-MSCs (17). The current results suggested that iPSC-MSCs presented a spindle-like morphology, which was consistent with a previous study on BMSCs (17). Moreover, the current results demonstrated that
iPSC-MSC attained $99 \%$ positive for CD73, 71\% positive for CD90 and $95 \%$ positive for CD105, which were typical cell markers comparable to BMSCs (25).

While iPSC-MSCs have similar surface markers as BMSCs, their gene expression profile differs (26), and iPSC-MSCs are genetically related to vascular progenitor cells. Additionally, iPSC-MSCs have similar osteogenic and chondrogenic differentiation properties as BMSCs, but their adipogenic differentiation potential is significantly lower compared with that of BMSCs $(12,26)$. Therefore, iPSC-MSCs 
are not entirely equivalent to BMSCs. Consistent with the aforementioned findings, the present results indicated that iPSC-MSCs had a satisfactory osteogenic and chondrogenic differentiation potential, which suggested that iPSC-MSCs are appropriate for bone tissue engineering.

At present, the role of DLX3 on cell proliferation has not been sufficiently investigated. A previous research group pointed out that stable overexpression of DLX3 gene inhibited the proliferation of human DPCs by inactivating the canonical Wnt pathway $(21,27)$. In the current study, a CCK-8 assay was used to assess the proliferation of iPSC-MSCs after transfection of DLX3 gene on days 1, 3, 5 and 7. Compared with the control group, the iPSC-MSC-DLX3 group demonstrated a significant decreased proliferative rate on days 5 and 7 . These results were consistent with the reported studies regarding human DPCs.

DLX3, expressed in osteo-/odontogenic lineages, is a crucial transcription factor for osteo-/odontogenic differentiation, mineralization and skeletal development (21,28-30). In BMSCs, transfection of DLX3 gene promotes the expression of ALP, Runt-related transcription factor 2 (RUNX2), OSX and OCN, as well as the formation of calcified matrix (20). Overexpression of DLX3 stimulates osteoprogenitor cells to express bone matrix proteins, such as COL-1, bone sialoprotein, OCN and ALP (9). Moreover, DLX3 is strongly expressed in differentiating and differentiated osteoblasts, and particularly upregulates OCN and COL-1 (31). An in vitro study also revealed that DLX3 could promote odontoblastic differentiation of human DPCs, and could increase the expression levels of ALP, DSPP, DMP1 and Nestin (21). However, one study provided the opposite evidence in vivo, and reported that neural crest deletion of DLX3 increased bone formation and mineralization in craniofacial bones, which suggested an inhibitory role for DLX3 in osteoblastic differentiation (32). RNA sequencing and chromatin immunoprecipitation-Seq analyses have further demonstrated that DLX3 regulates transcription factors crucial for bone formation, such as DLX5, DLX6, RUNX2 and Sp7, as well as genes important for mineral deposition (bone sialoprotein 2, ectonucleotide pyrophosphatase/phosphodiesterase family member 1 and matrix extracellular phosphoglycoprotein) and bone turnover (tumor necrosis factor receptor superfamily member 11B) (33). Furthermore, with the knockdown of DLX3, researchers have observed increased occupancy of DLX5, as well as enhanced and earlier occupancy of RUNX2 on the bone-specific osteocalcin promoter (33). Taken together, these findings provide evidence that DLX3 attenuates bone mass accrual to support bone homeostasis via osteogenic gene pathway regulation. In the present study, it was identified that DLX3 overexpression significantly upregulated osteogenic differentiation of iPSC-MSC by activating ALP, OCN, OPN and COL-1 in vitro. In addition, iPSC-MSC-DLX3 formed significantly more mineralized nodules compared with the control group. These results were consistent with a number of previous studies $(9,20,21,31)$.

Osteoporosis (OP) is a bone metabolic disease that is characterized by the degeneration of bone structure and decreased bone mass (34). OP occurs in $>1 / 3$ of women and $1 / 5$ of men $>50$ years old, and affects the health and lives of these individuals (35). The primary mechanism of OP is the dysregulation of the dynamic balance between bone formation and resorption, resulting in higher bone resorption than bone formation, which may lead to bone metabolism disorder (36). The present results suggested that DLX3 was a positive transcription factor in regulating osteogenic differentiation. Previous studies have reported that some osteogenic stimulators and transcription factors can induce the protein expression of DLX3. It has been revealed that DLX3 is a novel target of Estrogen receptor $\alpha$ (ER- $\alpha)$, and ER- $\alpha$ regulates osteoblast differentiation via the modulation of DLX3 expression and/or interaction with DLX3 (30). DLX3 is also a novel target of protein kinase A (PKA), and PKA mediates bone morphogenetic protein signaling during osteoblast differentiation, at least in part, by phosphorylating DLX3 and modulating the protein stability and function of DLX3 (37). The present results may facilitate the development of novel strategies for the targeted therapy of OP.

However, the present study has some limitations. It was assumed that multilineage induction experiments were enough to demonstrate the successful induction of human iPSCs into iPSC-MSCs, but the relative marker genes were not analyzed pre/post-osteogenic and chondrogenic induction. Therefore, it is hoped that these limitations can be improved in future studies.

In conclusion, the present study demonstrated that DLX3 exerted a positive role in regulating the osteogenesis of iPSC-MSCs. However, the specific underlying mechanism of DLX3 affecting osteogenic differentiation in iPSC-MSCs is yet to be fully elucidated. Further investigations, both in vitro and in vivo, are required to confirm its effect on osteogenic differentiation. Therefore, future studies will focus on the specific mechanism of DLX3 affecting osteogenic differentiation of iPSC-MSCs. Moreover, in vivo assays of transgenic DLX3 should be performed to further confirm its effect on osteogenesis.

\section{Acknowledgements}

Not applicable.

\section{Funding}

The present study was supported by the Guangdong Basic and Applied Basic Research Foundation (grant no. 2020A1515010239), National Natural Science Foundation of China (grant no. 81500825), Medical Scientific Research Foundation of Guangdong Province (grant no. A2015423), Special Fund for Public Welfare Research and Capacity Building of Guangdong Province (grant no. 2014A020212211), and Science and Technology Program of Guangzhou (grant no. 201607010205).

\section{Availability of data and materials}

The datasets used and/or analyzed during the current study are available from the corresponding author on reasonable request.

\section{Authors' contributions}

WZ and RL designed the study, prepared the figures and drafted the manuscript. JL and QL performed the experiments. 
YL conceptualized the study and drafted and revised the manuscript. All authors read and approved the final manuscript.

\section{Ethics approval and consent to participate}

The present study was approved by the Ethical Review Committee of Jinan University (approval no. 2015-045).

\section{Patient consent for publication}

Not applicable.

\section{Competing interests}

The authors declare that they have no competing interests.

\section{References}

1. Feng $X$ and McDonald JM: Disorders of bone remodeling. Annu Rev Pathol 6: 121-145, 2011.

2. Raisz LG: Pathogenesis of osteoporosis: Concepts, conflicts, and prospects. J Clin Invest 115: 3318-3325, 2005.

3. Cummings SR and Melton LJ: Epidemiology and outcomes of osteoporotic fractures. Lancet 359: 1761-1767, 2002.

4. Genant HK, Cooper C, Poor G, Reid I, Ehrlich G, Kanis J, Nordin BE, Barrett-Connor E, Black D, Bonjour JP, et al: Interim report and recommendations of the World Health Organization Task-Force for Osteoporosis. Osteoporos Int 10: 259-264, 1999.

5. Aspray TJ and Hill TR: Osteoporosis and the Ageing Skeleton. Subcell Biochem 91: 453-476, 2019.

6. Hassan MQ, Javed A, Morasso MI, Karlin J, Montecino M, van Wijnen AJ, Stein GS, Stein JL and Lian JB: Dlx3 transcriptional regulation of osteoblast differentiation: Temporal recruitment of Msx2, Dlx3, and Dlx5 homeodomain proteins to chromatin of the osteocalcin gene. Mol Cell Biol 24: 9248-9261, 2004.

7. Zhao N, Zeng L, Liu Y, Han D, Liu H, Xu J, Jiang Y, Li C, Cai T, Feng $\mathrm{H}$, et al: DLX3 promotes bone marrow mesenchymal stem cell proliferation through H19/miR-675 axis. Clin Sci (Lond) 131: 2721-2735, 2017

8. Li Y, Han D, Zhang H, Liu H, Wong S, Zhao N, Qiu L and Feng H: Morphological analyses and a novel de novo DLX3 mutation associated with tricho-dento-osseous syndrome in a Chinese family. Eur J Oral Sci 123: 228-234, 2015.

9. Choi SJ, Song IS, Ryu OH, Choi SW, Hart PS, Wu WW, Shen RF and Hart TC: A 4 bp deletion mutation in DLX3 enhances osteoblastic differentiation and bone formation in vitro. Bone 42: 162-171, 2008

10. Lin H, Sohn J, Shen H, Langhans MT and Tuan RS: Bone marrow mesenchymal stem cells: Aging and tissue engineering applications to enhance bone healing. Biomaterials 203: 96-110, 2019.

11. Sabapathy V and Kumar S: hiPSC-derived iMSCs: NextGen MSCs as an advanced therapeutically active cell resource for regenerative medicine. J Cell Mol Med 20: 1571-1588, 2016.

12. Kang R, Zhou Y, Tan S, Zhou G, Aagaard L, Xie L, Bünger C, Bolund L and Luo Y: Mesenchymal stem cells derived from human induced pluripotent stem cells retain adequate osteogenicity and chondrogenicity but less adipogenicity. Stem Cell Res Ther 6: 144, 2015.

13. Xie J, Peng C, Zhao Q, Wang X, Yuan H, Yang L, Li K, Lou X and Zhang Y: Osteogenic differentiation and bone regeneration of iPSC-MSCs supported by a biomimetic nanofibrous scaffold. Acta Biomater 29: 365-379, 2016.

14. Jungbluth P, Spitzhorn LS, Grassmann J, Tanner S, Latz D, Rahman MS, Bohndorf M, Wruck W, Sager M, Grotheer V, et al: Human iPSC-derived iMSCs improve bone regeneration in mini-pigs. Bone Res 7: 32, 2019.

15. Villa-Diaz LG, Brown SE, Liu Y, Ross AM, Lahann J, Parent JM and Krebsbach PH: Derivation of mesenchymal stem cells from human induced pluripotent stem cells cultured on synthetic substrates. Stem Cells 30: 1174-1181, 2012.

16. Liu Y, Goldberg AJ, Dennis JE, Gronowicz GA and Kuhn LT: One-step derivation of mesenchymal stem cell (MSC)-like cells from human pluripotent stem cells on a fibrillar collagen coating. PLoS One 7: e33225, 2012.
17. TheinHan WW, Liu J, Tang M, Chen W, Cheng L and Xu HH: Induced pluripotent stem cell-derived mesenchymal stem cell seeding on biofunctionalized calcium phosphate cements. Bone Res 1: 371-384, 2013.

18. Chen YS, Pelekanos RA, Ellis RL, Horne R, Wolvetang EJ and Fisk NM: Small molecule mesengenic induction of human induced pluripotent stem cells to generate mesenchymal stem/stromal cells. Stem Cells Transl Med 1: 83-95, 2012.

19. Meng X, Su RJ, Baylink DJ, Neises A, Kiroyan JB, Lee WY, Payne KJ, Gridley DS, Wang J, Lau KH, et al: Rapid and efficient reprogramming of human fetal and adult blood $\mathrm{CD} 34^{+}$cells into mesenchymal stem cells with a single factor. Cell Res 23: 658-672, 2013.

20. Sun S, Yu M, Fan Z, Yeh IT, Feng H, Liu H and Han D: DLX3 regulates osteogenic differentiation of bone marrow mesenchymal stem cells via Wnt/ $\beta$-catenin pathway mediated histone methylation of DKK4. Biochem Biophys Res Commun 516: 171-176, 2019.

21. Li X, Yang G and Fan M: Effects of homeobox gene distal-less 3 on proliferation and odontoblastic differentiation of human dental pulp cells. J Endod 38: 1504-1510, 2012.

22. Spandidos A, Wang X, Wang H and Seed B: PrimerBank: A resource of human and mouse PCR primer pairs for gene expression detection and quantification. Nucleic Acids Res 38 (Suppl 1): D792-D799, 2010.

23. Zhang W, Zhang X, Li J, Zheng J, Hu X, Xu M, Mao X and Ling J: Foxc2 and BMP2 induce osteogenic/odontogenic differentiation and mineralization of human stem cells from Apical Papilla. Stem Cells Int 2018: 2363917, 2018.

24. Livak KJ and Schmittgen TD: Analysis of relative gene expression data using real-time quantitative PCR and the 2(-Delta Delta C(T)) method. Methods 25: 402-408, 2001.

25. Zou L, Luo Y, Chen M, Wang G, Ding M, Petersen CC, Kang R, Dagnaes-Hansen F, Zeng Y, Lv N, et al: A simple method for deriving functional MSCs and applied for osteogenesis in 3D scaffolds. Sci Rep 3: 2243, 2013.

26. Xu M, Shaw G, Murphy M and Barry F: Induced pluripotent stem cell-derived mesenchymal stromal cells are functionally and genetically different from bone marrow-derived mesenchymal stromal cells. Stem Cells 37: 754-765, 2019.

27. Zhan Y, Li X, Gou X, Yuan G, Fan M and Yang G: Dlx3 inhibits the proliferation of human dental pulp cells through inactivation of canonical wnt/beta-catenin signaling pathway. Front Physiol 9: 1637,2018

28. Beanan MJ and Sargent TD: Regulation and function of Dlx3 in vertebrate development. Dev Dyn 218: 545-553, 2000.

29. Ghoul-Mazgar S, Hotton D, Lézot F, Blin-Wakkach C, Asselin A, Sautier JM and Berdal A: Expression pattern of Dlx3 during cell differentiation in mineralized tissues. Bone 37: 799-809, 2005.

30. Lee SH, Oh KN, Han Y, Choi YH and Lee KY: Estrogen Receptor $\alpha$ Regulates Dlx3-Mediated Osteoblast Differentiation. Mol Cells 39: 156-162, 2016.

31. Li H, Marijanovic I, Kronenberg MS, Erceg I, Stover ML, Velonis D, Mina M, Heinrich JG, Harris SE, Upholt WB, et al: Expression and function of Dlx genes in the osteoblast lineage. Dev Biol 316: 458-470, 2008.

32. Duverger O, Isaac J, Zah A, Hwang J, Berdal A, Lian JB and Morasso MI: In vivo impact of Dlx3 conditional inactivation in neural crest-derived craniofacial bones. J Cell Physiol 228: 654-664, 2013.

33. Isaac J, Erthal J, Gordon J, Duverger O, Sun HW, Lichtler AC, Stein GS, Lian JB and Morasso MI: DLX3 regulates bone mass by targeting genes supporting osteoblast differentiation and mineral homeostasis in vivo. Cell Death Differ 21: 1365-1376, 2014.

34. Sözen T, Özışık L and Başaran NC: An overview and management of osteoporosis. Eur J Rheumatol 4: 46-56, 2017.

35. Zou Z, Liu W, Cao L, Liu Y, He T, Peng S and Shuai C: Advances in the occurrence and biotherapy of osteoporosis. Biochem Soc Trans 48: 1623-1636, 2020.

36. Yin X, Zhou C, Li J, Liu R, Shi B, Yuan Q and Zou S: Autophagy in bone homeostasis and the onset of osteoporosis. Bone Res 7: 28, 2019.

37. Li H, Jeong HM, Choi YH, Kim JH, Choi JK, Yeo CY, Jeong HG, Jeong TC, Chun C and Lee KY: Protein kinase a phosphorylates Dlx 3 and regulates the function of Dlx3 during osteoblast differentiation. J Cell Biochem 115: 2004-2011, 2014.

This work is licensed under a Creative Commons Attribution-NonCommercial-NoDerivatives 4.0 International (CC BY-NC-ND 4.0) License. 